\title{
On a discrete West Nile epidemic model
}

\author{
SOPHIA R.-J. JANG \\ Department of Mathematics, University of Louisiana at Lafayette \\ Lafayette, LA 70504-1010 U.S.A. \\ E-mail: jang@louisiana.edu
}

\begin{abstract}
A West Nile epidemic model in discrete-time is proposed. The model consists of two interacting populations, the vector and the avian populations. The avian population is classified into susceptible, infective, and recovered classes while an individual vector is either susceptible or infective. The transmission of the disease is assumed only by mosquitoes bites and vertical transmission in the vector population. The model behavior depends on a lumped parameter $R_{0}$. The disease-free equilibrium is locally asymptotically stable if $R_{0}<1$. The system is uniformly persistent and possesses a unique endemic equilibrium if $R_{0}>1$. Consequently, the disease can persist in the populations if $R_{0}>1$.
\end{abstract}

Mathematical subject classification: 92D25, 92D40, 39A11.

Key words: West Nile virus, uniform persistence, Liapunov function.

\section{Introduction}

West Nile virus (WNV) is a kind of arthropod-borne virus that are maintained in nature through biological transmission between susceptible vertebrate hosts and blood-feeding arthropods such as mosquitoes. Vertebrates can become infected when an infected arthropod bites them to take a blood meal. The susceptible vectors then become infected once feed on an infected host.

WNV was first isolated from a woman in the West Nile District of Uganda in 1937 and has emerged in recent years in many regions of the United States and Canada. The disease presents a threat and challenge to public and animal health. West Nile virus has been detected in dead birds of at least 138 
species. Although birds, particularly crows and jays, infected with the virus can die or become ill, most infected birds do survive. We refer the reader to www.cdc.gov/ncidod/dvbid/westnile for more information about the virus history and its ecology.

Since data collected for the West Nile virus are usually discrete, we develop a discrete-time West-Nile model to investigate evolution of the disease between mosquitoes and bird reservoir hosts. Discrete time West Nile models have been studied in $[15,17]$. However, our modeling assumptions are different from that given in $[15,17]$. In [15] the vector population is partitioned into larval, susceptible, exposed, and infective classes, and all the newborns are in the larval class, while in [17] the vector population also has an exposed compartment and there is no vertical transmission. Moreover, our incidence rate is different from that studied in $[15,17]$. Our model derivation is based on a recent continuoustime model proposed by Cruz-Pacheco et al. [5]. Although other vertebrates such as horses and humans do become infected, these populations are not modeled here.

The resulting epidemic model is a four-dimensional system of difference equations. Sufficient conditions for which solutions remain nonnegative are derived. It is shown that the disease-free equilibrium always exists. Its stability depends on a threshold $R_{0}$. The disease-free equilibrium is locally asymptotically stable if $R_{0}<1$ and unstable if $R_{0}>1$. There exists a unique endemic equilibrium and the system is uniformly persistent when $R_{0}>1$. Consequently, the WNV can persist if $R_{0}>1$. When there is no disease related death for the avian population, it can be shown that the disease-free equilibrium is globally asymptotically stable for $R_{0} \leq 1$.

In the following section, model derivation will be presented. Section 3 provides stability analysis of the model. Numerical simulations and a brief summary are given in the last section.

\section{Model derivation}

Our model consists of two interacting populations: birds and mosquitoes. The transmission of the disease is only by mosquitoes bites and vertical transmission in the vector population. Let $N_{a}(t)$ and $N_{v}(t)$ denote the avian and vector pop- 
ulations at time $t$, respectively for $t=0,1, \ldots$ We assume that the mosquito population under the period of study is a constant, $N_{v}$, and the bird population has a constant recruitment rate $\Lambda_{a}$ per unit time due to birth and immigration. However, the new arrival birds are all susceptible. The death rates of avian and vector populations are denoted by $\mu_{a}$ and $\mu_{v}$, respectively.

For simplicity, the birth rate of the vector population is $\mu_{v}$ which is the same as its death rate. That is, $\mu_{v}$ is the number of births per individual per unit time for the mosquito population. It is also assumed that the bird population in the absence of the disease is governed by the difference equation $N_{a}(t+1)=$ $\Lambda_{a}+\left(1-\mu_{a}\right) N_{a}(t)$. As a result, the bird population in the absence of the disease will always stabilize at the level $\left(\Lambda_{a}\right) /\left(\mu_{a}\right)$.

Similar to the idea used by Kermack and McKendrick [12] for modeling epidemics, the avian population at time $t$ is separated into three compartments: susceptible $S_{a}(t)$, the healthy susceptible individuals who can contract the disease, infectives $I_{a}(t)$, the individuals who are infected and are infectious, and recovered $R_{a}(t)$, who are cured. That is, $N_{a}(t)=S_{a}(t)+I_{a}(t)+R_{a}(t)$ for $t \geq 0$. Since mosquitos have short life span, the vector population at any given time $t$ is only classified into susceptible, $S_{v}(t)$, and infectives, $I_{v}(t)$. There is no recovered class for the vector population and $S_{v}(t)+I_{v}(t)=N_{v}>0$ for $t \geq 0$.

Let $b$ be the average number of bites per mosquito per unit time. The transmission probability from vectors to birds and from birds to vectors are constants and denoted by $\beta_{a}$ and $\beta_{v}$, respectively. Hence a bird receives on average $b \frac{N_{v}}{N_{a}}$ bites per unit time. Therefore the infection rate per susceptible bird is

$$
b \beta_{a} \frac{N_{v}}{N_{a}} \frac{I_{v}}{N_{v}}=b \beta_{a} \frac{I_{v}}{N_{a}}
$$

and the infection rate per susceptible mosquito is

$$
b \beta_{v} \frac{I_{a}}{N_{a}} .
$$

We assume that the infected birds are recovered at a constant rate $\gamma_{a}$, and let $\alpha_{a}$ be the disease related death rate for the avian population. From the data given in $[13,16]$ (cf. [5]) it is reasonable to assume that

(H1) $\alpha_{a} \leq \gamma_{a}$.

Comp. Appl. Math., Vol. 26, N. 3, 2007 
We remark that assumption (H1) may not be satisfied for some spices of birds such as American crow and blue jay. However, many other species of birds such as common grackle, house sparrow, European starling etc. do have small WNV mortality ([13]), and consequently (H1) will fit in to these particular species of birds.

Notice the average infectious period for an infected bird is

$$
\frac{1}{\gamma_{a}+\mu_{a}+\alpha_{a}} \text {. }
$$

Furthermore, it is assumed that all these parameters $\Lambda_{a}, \mu_{a}, \mu_{v}, b, \beta_{a}, \beta_{v}, N_{v}, \gamma_{a}$ and $\alpha_{a}$ are positive. Since vertical transmission of the vector population has been found as an important mechanism in maintaining the virus in natural populations $[3,7,10]$, we assume a constant fraction $p, 0 \leq p \leq 1$, of the offspring of the infectious vectors is infectious. Under these biological assumptions, the interaction between vector and avian populations are given below:

$$
\left\{\begin{array}{l}
S_{a}(t+1)=\Lambda_{a}+\left(1-\mu_{a}\right) S_{a}(t)-\frac{b \beta_{a}}{N_{a}(t)} I_{v}(t) S_{a}(t) \\
I_{a}(t+1)=\frac{b \beta_{a}}{N_{a}(t)} I_{v}(t) S_{a}(t)+\left(1-\gamma_{a}-\mu_{a}-\alpha_{a}\right) I_{a}(t) \\
R_{a}(t+1)=\left(1-\mu_{a}\right) R_{a}(t)+\gamma_{a} I_{a}(t) \\
N_{a}(t+1)=\Lambda_{a}+\left(1-\mu_{a}\right) N_{a}(t)-\alpha_{a} I_{a}(t) \\
S_{v}(t+1)=S_{v}(t)+(1-p) \mu_{v} I_{v}(t)-\frac{b \beta_{v}}{N_{a}(t)} I_{a}(t) S_{v}(t) \\
I_{v}(t+1)=\left(1-\mu_{v}\right) I_{v}(t)+p \mu_{v} I_{v}(t)+\frac{b \beta_{v}}{N_{a}(t)} I_{a}(t) S_{v}(t) \\
S_{a}(0), I_{a}(0), R_{a}(0), S_{v}(0), I_{v}(0) \geq 0, N_{a}(0)>0 .
\end{array}\right.
$$

Notice as the birth and death rates of the vector population are the same and offsprings of susceptible mosquitoes are born susceptible, the equation for $S_{v}$ has the above form.

Discrete time epidemic models have been studied by Allen [1, 2], Lewis et al. [15], Thomas and Urena [17], and more recently by Franke [9] on periodic epidemic models. In $[1,2]$, the models are expressed in terms of time unit $\Delta t$ 
and nonnegativity of the solutions are derived using the quantity $\Delta t$ and model parameters. Since data for the West Nile epidemics given in the literature $[3,5$, $7,10,16]$ are in terms of days, our time unit is taken to be a day and model (2.1) does not involve time unit $\Delta t$ as in [1,2]. We now impose the following conditions on the parameters so that solutions of (2.1) will remain nonnegative as shown in Proposition 2.1.

$$
b \beta_{a} N_{v} \leq \Lambda_{a}, \gamma_{a}+\mu_{a}+\alpha_{a} \leq 1, b \beta_{v} \leq 1, \text { and } \mu_{v} \leq 1
$$

We remark that the first three conditions in (H2) imposed on the parameters are reasonable restrictions. For example, since the time unit is taken to be one day, then according to the data given in $[5,16]$, we have $\beta_{a}=1, b=0.75$, and the maximum values of $\beta_{v}$ is 0.68 , of $\gamma_{a}$ is 0.36 , of $\alpha_{a}$ is 0.19 , of $\mu_{a}$ is 0.0004 , and of $\mu_{v}$ is 0.06 for a variety of bird species such as blue jay, common grackle, American crow, house sparrow, American robin, rock dove etc. and different species of mosquitos. Therefore the first three conditions in (H2) are easily satisfied. However, we would need the total population of vector to be small or the new arrival of birds to be large in our study for the last inequality in (H2) to be true.

Proposition 2.1. Solutions of system (2.1) remain nonnegative and are bounded.

Proof. Let $\left(S_{a}(t), I_{a}(t), R_{a}(t), N_{a}(t), S_{v}(t), I_{v}(t)\right)$ be a solution of (2.1) with $S_{a}(0), I_{a}(0), R_{a}(0), S_{v}(0), I_{v}(0) \geq 0$ and $N_{a}(0)>0$. It is sufficient to prove nonnegativity for $t=1$. Since $S_{a}(0)+I_{a}(0)+R_{a}(0)=N_{a}(0)>0$ and $S_{v}(0)+I_{v}(0)=N_{v}>0, S_{a}(1) \geq \Lambda_{a}+\left(1-\mu_{a}\right) S_{a}(0)-b \beta_{a} I_{v}(0) \geq \Lambda_{v}+$ $\left(1-\mu_{a}\right) S_{a}(0)-b \beta_{a} N_{v} \geq\left(1-\mu_{a}\right) S_{a}(0) \geq 0$ by (H2) and (H3). It is clear that $I_{a}(1), R_{a}(1) \geq 0$ by (H3). Moreover, $S_{v}(1) \geq S_{v}(0)+(1-p) \mu_{v} I_{v}(0)-$ $b \beta_{v} S_{v}(0) \geq 0$ by (H4). Similarly, $N_{a}(1) \geq \Lambda_{a}+\left(1-\mu_{a}-\alpha_{a}\right) N_{a}(0) \geq \Lambda_{a}$ and $I_{v}(1) \geq 0$ by (H3) and (H5), respectively. Therefore, solutions of (2.1) remain nonnegative by induction.

Notice $N_{a}(t+1) \leq \Lambda_{a}+\left(1-\mu_{a}\right) N_{a}(t)$ for $t \geq 0$ implies

$$
\limsup _{t \rightarrow \infty} N_{a}(t) \leq \frac{\Lambda_{a}}{\mu_{a}} \text {. }
$$

Comp. Appl. Math., Vol. 26, N. 3, 2007 
As $S_{a}(t), I_{a}(t), R_{a}(t)$ are nonnegative and satisfy $S_{a}(t)+I_{a}(t)+R_{a}(t)=N_{a}(t)$ for $t \geq 0$, we have

$$
\limsup _{t \rightarrow \infty} S_{a}(t) \leq \frac{\Lambda_{a}}{\mu_{a}}, \quad \limsup _{t \rightarrow \infty} I_{a}(t) \leq \frac{\Lambda_{a}}{\mu_{a}}, \quad \text { and } \quad \limsup _{t \rightarrow \infty} R_{a}(t) \leq \frac{\Lambda_{a}}{\mu_{a}} .
$$

Moreover, since $S_{v}(t+1)+I_{v}(t+1)=N_{v}$ for $t \geq 0$ and solutions remain nonnegative, $S_{v}(t), I_{v}(t) \leq N_{v}$ for $t \geq 0$. Therefore, solutions of (2.1) are bounded.

It follows from the proof of Proposition 2.1 that $N_{a}(t) \geq \Lambda_{a}$ for $t \geq 1$ if $N_{a}(0)>0$. Therefore system (2.1) is well-defined. Furthermore, since $I_{a}(t)+S_{a}(t)+R_{a}(t)=N_{a}(t)$ and $S_{v}(t)+I_{v}(t)=N_{v}$ for $t \geq 0$ from modeling assumptions, we are able to reduce the dimension of system (2.1) so that system (2.1) is equivalent to the following four-dimensional system of difference equations

$$
\left\{\begin{array}{l}
S_{a}(t+1)=\Lambda_{a}+\left(1-\mu_{a}\right) S_{a}(t)-\frac{b \beta_{a}}{N_{a}(t)} I_{v}(t) S_{a}(t) \\
I_{a}(t+1)=\frac{b \beta_{a}}{N_{a}(t)} I_{v}(t) S_{a}(t)+\left(1-\gamma_{a}-\mu_{a}-\alpha_{a}\right) I_{a}(t) \\
N_{a}(t+1)=\Lambda_{a}+\left(1-\mu_{a}\right) N_{a}(t)-\alpha_{a} I_{a}(t) \\
I_{v}(t+1)=\left(1-\mu_{v}\right) I_{v}(t)+p \mu_{v} I_{v}(t)+\frac{b \beta_{v}}{N_{a}(t)} I_{a}(t)\left(N_{v}-I_{v}(t)\right) \\
S_{a}(0), I_{a}(0), I_{v}(0) \geq 0, N_{a}(0)>0 .
\end{array}\right.
$$

\section{Mathematical analysis}

We first study the existence of steady state solutions of (2.2). Clearly there always exists a trivial steady state $E_{0}=\left(\frac{\Lambda_{a}}{\mu_{a}}, 0, \frac{\Lambda_{a}}{\mu_{a}}, 0\right)$, the disease-free equilibrium. The Jacobian matrix of (2.2) evaluated at $E_{0}$ has the following form

$$
J\left(E_{0}\right)=\left(\begin{array}{cccc}
1-\mu_{a} & 0 & 0 & -b \beta_{a} \\
0 & 1-\gamma_{a}-\mu_{a}-\alpha_{a} & 0 & b \beta_{a} \\
0 & -\alpha_{a} & 1-\mu_{a} & 0 \\
0 & \mu_{a} b \beta_{v} N_{v} / \Lambda_{a} & 0 & 1-(1-p) \mu_{v}
\end{array}\right) .
$$


Let $J_{1}$ be the lower $3 \times 3$ submatrix of $J\left(E_{0}\right)$. Then $J_{1}$ is similar to

$$
\left(\begin{array}{ccc}
1-\gamma_{a}-\mu_{a}-\alpha_{a} & b \beta_{a} & 0 \\
\mu_{a} b \beta_{v} N_{v} / \Lambda_{a} & 1-(1-p) \mu_{v} & 0 \\
-\alpha_{a} & 0 & 1-\mu_{a}
\end{array}\right) .
$$

Therefore eigenvalues of $J\left(E_{0}\right)$ are $1-\mu_{a}$ of multiplicity 2 and eigenvalues of

$$
J_{2}=\left(\begin{array}{cc}
1-\gamma_{a}-\mu_{a}-\alpha_{a} & b \beta_{a} \\
\mu_{a} b \beta_{v} N_{v} / \Lambda_{a} & 1-(1-p) \mu_{v}
\end{array}\right) .
$$

Notice

$$
\operatorname{tr} J_{2}=2-\gamma_{a}-\mu_{a}-\alpha_{a}-(1-p) \mu_{v}
$$

and

$$
\operatorname{det} J_{2}=\left(1-\gamma_{a}-\mu_{a}-\alpha_{a}\right)\left[1-(1-p) \mu_{v}\right]-\frac{b^{2} \mu_{a} N_{v} \beta_{v} \beta_{a}}{\Lambda_{a}} .
$$

Jury conditions imply that eigenvalues $\lambda$ of $J_{2}$ satisfy $|\lambda|<1$ if and only if $\left|\operatorname{tr} J_{2}\right|<1+\operatorname{det} J_{2}<2$ [8]. It follows from (H2) that $\operatorname{tr} J_{2}>0$ and thus we need to verify $\operatorname{tr} J_{2}<1+\operatorname{det} J_{2}<2$ for the local stability of $E_{0}$.

Notice det $J_{2}<1$ is trivially true and thus $1+\operatorname{det} J_{2}<2$ holds. To verify $\operatorname{tr} J_{2}<1+\operatorname{det} J_{2}$ we shall separate our discussion into two cases: $0 \leq p<1$ and $p=1$. When $0 \leq p<1$, a simple computation yields

$$
\operatorname{tr} J_{2}<1+\operatorname{det} J_{2} \text { if and only if } \frac{b^{2} \mu_{a} N_{v} \beta_{a} \beta_{v}}{\Lambda_{a} \mu_{v}(1-p)\left(\gamma_{a}+\mu_{a}+\alpha_{a}\right)}<1 .
$$

Let

$$
R_{0}=\frac{b^{2} \mu_{a} N_{v} \beta_{a} \beta_{v}}{\Lambda_{a} \mu_{v}(1-p)\left(\gamma_{a}+\mu_{a}+\alpha_{a}\right)} .
$$

It follows that $E_{0}$ is locally asymptotically stable if $R_{0}<1$, and unstable if $R_{0}>1$. When $p=1$,

$\operatorname{tr} J_{2}=2-\gamma_{a}-\mu_{a}-\alpha_{a} \quad$ and $\quad \operatorname{det} J_{2}=1-\gamma_{a}-\mu_{a}-\alpha_{a}-\frac{b^{2} \mu_{a} N_{v} \beta_{v} \beta_{a}}{\Lambda_{a}}$.

Therefore $\operatorname{tr} J_{2}<1+\operatorname{det} J_{2}$ if and only if

$$
2-\gamma_{a}-\mu_{a}-\alpha_{a}<2-\gamma_{a}-\mu_{a}-\alpha_{a}-\frac{b^{2} \mu_{a} N_{v} \beta_{v} \beta_{a}}{\Lambda_{a}} .
$$


The above inequality is never valid. Therefore $E_{0}$ is always unstable when $p=1$.

Observe that $\frac{b \mu_{a} N_{v} \beta_{v}}{\Lambda_{a}\left(\gamma_{a}+\mu_{a}+\alpha_{a}\right)}$ can be interpreted as the number of infections produced by a single infected bird during its infectious period in a susceptible mosquito population when the avian population is stabilized at the population level $\Lambda_{a} / \mu_{a}$. Similarly, $\frac{b \beta_{a}}{(1-p) \mu_{v}}$ is the number of infections produced by a single infectious mosquito during its lifetime in a susceptible avian population. Therefore, $\sqrt{R_{0}}$, the geometric mean of these two quantities, may be regarded as the basic reproductive number of the disease.

We proceed to examine the existence of an interior steady state. An interior steady state $\left(\bar{S}_{a}, \bar{I}_{a}, \bar{N}_{a}, \bar{I}_{v}\right)$ must satisfy

$$
\left\{\begin{array}{l}
\Lambda_{a}-\mu_{a} \bar{S}_{a}-\frac{b \beta_{a}}{\bar{N}_{a}} \bar{I}_{v} \bar{S}_{a}=0 \\
\frac{b \beta_{a}}{\bar{N}_{a}} \bar{I}_{v} \bar{S}_{a}-\left(\gamma_{a}+\mu_{a}+\alpha_{a}\right) \bar{I}_{a}=0 \\
\Lambda_{a}-\mu_{a} \bar{N}_{a}-\alpha_{a} \bar{I}_{a}=0 \\
-\mu_{v} \bar{I}_{v}+p \mu_{v} \bar{I}_{v}+\frac{b \beta_{v}}{\bar{N}_{a}} \bar{I}_{a}\left(N_{v}-\bar{I}_{v}\right)=0 .
\end{array}\right.
$$

Adding the first two equations of (3.5) resulting

$$
\Lambda_{a}-\mu_{a} \bar{S}_{a}-\left(\gamma_{a}+\mu_{a}+\alpha_{a}\right) \bar{I}_{a}=0 .
$$

Let

$$
A=\gamma_{a}+\mu_{a}+\alpha_{a}
$$

Then

$$
\bar{S}_{a}=\frac{\Lambda_{a}-A \bar{I}_{a}}{\mu_{a}},
$$

and $\bar{S}_{a}>0$ if and only if $\bar{I}_{a}<\frac{\Lambda_{a}}{A}$. The third and fourth equilibrium equations imply

$$
\bar{N}_{a}=\frac{\Lambda_{a}-\alpha_{a} \bar{I}_{a}}{\mu_{a}}
$$

and

$$
\bar{I}_{v}=\frac{b \beta_{v} \mu_{a} N_{v} \bar{I}_{a}}{\left(\Lambda_{a}-\alpha_{a} \bar{I}_{a}\right)(1-p) \mu_{v}+\mu_{a} b \beta_{v} \bar{I}_{a}} .
$$


Substituting these into the second equilibrium equation we have for $0 \leq p<1$, $\bar{I}_{a}>0$ must satisfy

$$
\hat{A} x^{2}+\hat{B} x+\hat{C}=0
$$

where

$$
\begin{gathered}
\hat{A}=\alpha_{a}\left[b \mu_{a} \beta_{v}-(1-p) \alpha_{a} \mu_{v}\right], \\
\hat{B}=2 \Lambda_{a}(1-p) \mu_{v} \alpha_{a}-b \Lambda_{a} \mu_{a} \beta_{v}-(1-p) A \Lambda_{a} \mu_{v} R_{0}, \quad \text { and } \\
\hat{C}=\Lambda_{a}^{2} \mu_{v}(1-p)\left(R_{0}-1\right) .
\end{gathered}
$$

Let

$$
f(x)=\hat{A} x^{2}+\hat{B} x+\hat{C} .
$$

Notice

$$
f(0)=\hat{C}
$$

and since $A=\alpha_{a}+\gamma_{a}+\mu_{a}$,

$$
f\left(\frac{\Lambda_{a}}{A}\right)=\frac{1}{A^{2}}\left[b \Lambda_{a}^{2} \mu_{a} \beta_{v}\left(\alpha_{a}-A\right)-(1-p) \Lambda_{a}^{2} \mu_{v}\left(A-\alpha_{a}\right)^{2}\right]<0 .
$$

When $R_{0}<1, \hat{C}<0$ and hence for $f(x)$ to have at least one positive root in $\left(0, \Lambda_{a} / A\right)$ it is necessary that

$$
\hat{A}<0<\frac{-\hat{B}}{2 \hat{A}}<\frac{\Lambda_{a}}{A} \quad \text { and } \quad \hat{B}^{2}-4 \hat{A} \hat{C}>0
$$

Notice that the last inequality is equivalent to $\frac{-\hat{B}}{2 \hat{A}}>\frac{-2 \hat{C}}{\hat{B}}$, and

$$
\frac{-2 \hat{C}}{\hat{B}}>\frac{\Lambda_{a}}{A}
$$

if and only if

$$
b \mu_{a} \beta_{v}-A \mu_{v}(1-p) R_{0}+2\left(\mu_{a}+\gamma_{a}\right) \mu_{v}(1-p)>0 .
$$

Substituting $R_{0}$ by the expression (3.4) and using (H2), one can see that the above inequality is trivially true. Therefore there exists no feasible solution $\bar{I}_{a}$ 
for (3.6) in $\left(0, \Lambda_{a} / A\right)$ if $0 \leq p<1$ and $R_{0}<1$. Consequently, system (2.2) has no interior steady state if $0 \leq p<1$ and $R_{0}<1$.

On the other hand if $R_{0}>1$ then since $\hat{C}>0$ and $f\left(\Lambda_{a} / A\right)<0$, it is clear that (3.6) has a unique solution $\bar{I}_{a} \in\left(0, \Lambda_{a} / A\right)$. As a result, system (2.2) has a unique endemic equilibrium $E_{1}=\left(\bar{S}_{a}, \bar{I}_{a}, \bar{N}_{a}, \bar{I}_{v}\right)$ if $0 \leq p<1$ and $R_{0}>1$. If $0 \leq p<1$ and $R_{0}=1$, then $\hat{C}=0$ and $f(x)=0$ has solutions 0 and $-\hat{B} / \hat{A}$. Notice $\hat{B}=\Lambda_{a}(1-p) \mu_{v} \alpha_{a}-(1-p)\left(\mu_{a}+\gamma_{a}\right) \Lambda_{a} \mu_{v}-b \Lambda_{a} \mu_{a} \beta_{v}<0$ by (H1). If $\hat{A}>0$ then it is straightforward to show that $-\hat{B} / \hat{A}>\Lambda_{a} / A$, and if $\hat{A}<0$ then it is trivial that $-\hat{B} / \hat{A}<0$. Therefore if $0 \leq p<1$ and $R_{0}=1$, there is no feasible $\bar{I}_{a}$. We conclude that system (2.2) has no interior steady state if $0 \leq p<1$ and $R_{0} \leq 1$, and has a unique endemic equilibrium if $R_{0}>1$.

When $p=1$, the threshold $R_{0}$ in (3.4) is not defined and the $I_{a}$-component, $\bar{I}_{a}>0$, of an interior steady state must satisfy (3.6) with

$$
\hat{A}=\alpha_{a} b \mu_{a} \beta_{v}, \hat{C}=\frac{b^{2} \beta_{a} \beta_{v} \mu_{a} N_{v} \Lambda_{a}}{A}
$$

and

$$
\hat{B}=-b \Lambda_{a} \mu_{a} \beta_{v}-b^{2} \beta_{a} \beta_{v} \mu_{a} N_{v} .
$$

Since $\hat{A}>0, \hat{C}>0$ and $f\left(\Lambda_{a} / A\right)<0, f(x)=0$ has a unique solution $\bar{I}_{a}$ in $\left(0, \Lambda_{a} / A\right)$. Consequently, $(2.2)$ has a unique endemic equilibrium $E_{1}=$ $\left(\bar{S}_{a}, \bar{I}_{a}, \bar{N}_{a}, \bar{I}_{v}\right)$ when $p=1$. Recall in this case that the disease-free equilibrium $E_{0}=\left(\Lambda_{a} / \mu_{a}, 0, \Lambda_{a} / \mu_{a}, 0\right)$ is unstable. The above discussion is summarized below.

Proposition 3.1. If $0 \leq p<1$ and $R_{0} \leq 1$, then $E_{0}=\left(\Lambda_{a} / \mu_{a}, 0, \Lambda_{a} / \mu_{a}, 0\right)$ is the only equilibrium and $E_{0}$ is locally asymptotically stable if $R_{0}<1$. If $0 \leq p<1$ and $R_{0}>1$, then $E_{0}$ is unstable and system (2.2) has a unique interior steady state $E_{1}=\left(\bar{S}_{a}, \bar{I}_{a}, \bar{N}_{a}, \bar{I}_{v}\right)$. If $p=1$, then $E_{0}$ is unstable and $E_{1}$ exists for (2.2).

Our next goal is to determine local stability of the steady state $E_{1}$. When $p=1$, it follows from (3.5) that $\bar{I}_{v}=N_{v}$. Therefore the Jacobian matrix of 
system (2.2) evaluated at $E_{1}$ has the following form

$$
J\left(E_{1}\right)=\left(\begin{array}{cccc}
1-\mu_{a}-b \beta_{a} N_{v} / \bar{N}_{a} & 0 & b \beta_{a} N_{v} \bar{S}_{a} / \bar{N}_{a}^{2} & -b \beta_{a} \bar{S}_{a} / \bar{N}_{a} \\
b \beta_{a} N_{v} / \bar{N}_{a} & 1-A & -b \beta_{a} N_{v} \bar{S}_{a} / \bar{N}_{a}^{2} & b \beta_{a} \bar{S}_{a} / \bar{N}_{a} \\
0 & -\alpha_{a} & 1-\mu_{a} & 0 \\
0 & 0 & 0 & 1-b \beta_{v} \bar{I}_{a} / \bar{N}_{a}
\end{array}\right) .
$$

Clearly $1-b \beta_{v} \bar{I}_{a} / \bar{N}_{a}$ is an eigenvalue of $J\left(E_{1}\right)$ which is less than 1 but greater than zero by (H4). The upper $3 \times 3$ submatrix of $J\left(E_{1}\right)-\lambda I$ is similar to the following matrix

$$
\hat{J}=\left(\begin{array}{ccc}
1-\mu_{a}-\lambda & 1-A-\lambda & 0 \\
b \beta_{a} N_{v} / \bar{N}_{a} & 1-A-\lambda & -b \beta_{a} N_{v} \bar{S}_{a} / \bar{N}_{a}^{2} \\
0 & -\alpha_{a} & 1-\mu_{a}-\lambda
\end{array}\right) .
$$

Using the third row expansion we see that $1-\mu_{a}$ is another eigenvalue and the rest of the two eigenvalues satisfy

$$
\lambda^{2}+\operatorname{tr} \bar{J} \lambda+\operatorname{det} \bar{J}=0
$$

where

$$
\operatorname{tr} \bar{J}=b \beta_{a} N_{v} / \bar{N}_{a}-2+\mu_{a}+A
$$

and

$$
\operatorname{det} \bar{J}=(1-A)\left(1-\mu_{a}\right)-b \alpha_{a} \beta_{a} N_{v} \bar{S}_{a} / \bar{N}_{a}^{2}-(1-A) b \beta_{a} N_{v} / \bar{N}_{a} .
$$

Since det $\hat{J}<1$, applying the Jury conditions, we need to very that $-1-\operatorname{det} \bar{J}<$ $\operatorname{tr} \bar{J}<1+\operatorname{det} \bar{J}$. A straightforward calculation yields $\operatorname{tr} \bar{J}<1+\operatorname{det} \bar{J}$ if and only if

$$
A \mu_{a}+\frac{A b \beta_{a} N_{v}}{\bar{N}_{a}}-\frac{b \alpha_{a} \beta_{a} \bar{S}_{a} N_{v}}{\bar{N}_{a}^{2}}>0 .
$$

Since $\bar{S}_{a}<\bar{N}_{a}$ and $A>\alpha_{a}$, the above inequality is clearly true. Moreover, $-1-\operatorname{det} \bar{J}<\operatorname{tr} \bar{J}$ if and only if

$$
-\mu_{a} A+(1-A) \frac{b \beta_{a} N_{v}}{\bar{N}_{a}}+\frac{b \alpha_{a} \beta_{a} N_{v} \bar{S}_{a}}{\bar{N}_{a}^{2}}<\frac{b \beta_{a} N_{v}}{\bar{N}_{a}} .
$$

This inequality also holds as $A>\alpha_{a}$ and $\bar{S}_{a}<\bar{N}_{a}$. We now summarize our discussion in the following proposition. 
Proposition 3.2. System (2.2) has steady states $E_{0}=\left(\Lambda_{a} / \mu_{a}, 0, \Lambda_{a} / \mu_{a}, 0\right)$ and $E_{1}=\left(\bar{S}_{a}, \bar{I}_{a}, \bar{N}_{a}, \bar{I}_{v}\right)$ when $p=1$, where $E_{0}$ is unstable and $E_{1}$ is locally asymptotically stable.

It is not easy to verify whether the endemic-equilibrium $E_{1}$ is locally asymptotically stable when $0 \leq p<1$ and $R_{0}>1$. We show that the disease can persist by showing that the system is uniformly persistent. We first briefly discuss terminology used in Hofbauer and So [11] which will be adopted for our analysis. Let $(X, d)$ be a metric space and $h: X \rightarrow X$ be continuous with a closed subspace $Y$ such that $X \backslash Y$ is forward invariant under $h$. It is assumed that $X$ has a global attractor $\mathcal{A}$. Let $M$ be the maximal compact invariant set in $Y$. Then $h$ is uniformly persistent (with respect to $Y$ ) i.e., there exists $m>0$ such that $\lim \inf _{t \rightarrow \infty} d\left(h^{t}(x), Y\right)>m$ for all $x \in X \backslash Y$ if and only if $M$ is isolated in $\mathcal{A}$ and $W^{s}(M)=\left\{x \in X: h^{t}(x) \rightarrow M\right.$ as $\left.t \rightarrow \infty\right\} \subset Y$ [11, Theorem 4.1].

Theorem 3.3. System (2.2) is uniformly persistent if either $0 \leq p<1$ and $R_{0}>1$ or if $p=1$.

Proof. Let $X=\mathbb{R}_{+}^{4}$ and $Y=\partial \mathbb{R}_{+}^{4}$, the boundary of $X$. Let $H$ denote the map induced by system (2.2). It follows from the proof of Proposition 2.1 that $S_{a}(t), I_{a}(t), N_{a}(t), I_{v}(t)>0$ for $t \geq 1$ if the initial condition is positive. Therefore $X \backslash Y$ is positively invariant for system (2.2). Clearly system (2.2) has a global attractor and the only invariant set in $Y$ is $\left\{E_{0}\right\}$, which is moreover isolated in $\left\{\left(S_{a}, I_{a}, N_{a}, I_{v}\right) \in \mathbb{R}_{+}^{4}: S_{a}+I_{a} \leq \Lambda_{a} / \mu_{a}, N_{a} \leq \Lambda_{a} / \mu_{a}, I_{v} \leq N_{v}\right\}$.

To show $W^{s}\left(\left\{E_{0}\right\}\right) \subset Y$, suppose on the contrary that there exists a solution $\left(S_{a}(t), I_{a}(t), N_{a}(t), I_{v}(t)\right)$ with $S_{a}(0)>0, I_{a}(0)>0, N_{a}(0)>0$, and $I_{v}(0)>$ 0 such that $\lim _{t \rightarrow \infty} S_{a}(t)=\lim _{t \rightarrow \infty} N_{a}(t)=\Lambda_{a} / \mu_{a}$ and $\lim _{t \rightarrow \infty} I_{a}(t)=$ $\lim _{t \rightarrow \infty} I_{v}(t)=0$. Then for any $\epsilon>0$ there exists $t_{0}>0$ such that

$$
1-\epsilon<\frac{S_{a}(t)}{N_{a}(t)}<1+\epsilon, 0<I_{a}(t), I_{v}(t)<\epsilon, \text { and } N_{a}(t)<\Lambda_{a} / \mu_{a}+\epsilon
$$

for $t \geq t_{0}$. We first consider the case when $0 \leq p<1$ and $R_{0}>1$. Since $R_{0}>1$, we can choose $\epsilon>0$ such that

$$
\frac{b^{2} \mu_{a} \beta_{a} \beta_{v}\left(N_{v}-\epsilon\right)(1-\epsilon)}{\left(\Lambda_{a}+\epsilon \mu_{a}\right) \mu_{v}(1-p)\left(\gamma_{a}+\mu_{a}+\alpha_{a}\right)}>1 .
$$


We have by system (2.2) that

$$
\begin{gathered}
I_{a}(t+1) \geq b \beta_{a}(1-\epsilon) I_{v}(t)+\left(1-\gamma_{a}-\mu_{a}-\alpha_{a}\right) I_{a}(t) \\
I_{v}(t+1) \geq \frac{b \beta_{v}}{\Lambda_{a} / \mu_{a}+\epsilon}\left(N_{v}-\epsilon\right) I_{a}(t)+\left[1-(1-p) \mu_{v}\right] I_{v}(t)
\end{gathered}
$$

for $t \geq t_{0}$. Consider the following linear system

$$
\left\{\begin{array}{l}
x(t+1)=\left(1-\gamma_{a}-\mu_{a}-\alpha_{a}\right) x(t)+b \beta_{a}(1-\epsilon) y(t) \\
y(t+1)=\frac{b \beta_{v} \mu_{a}}{\Lambda_{a}+\epsilon \mu_{a}}\left(N_{v}-\epsilon\right) x(t)+\left[1-(1-p) \mu_{v}\right] y(t) \\
x\left(t_{0}\right)=I_{a}\left(t_{0}\right), y\left(t_{0}\right)=I_{v}\left(t_{0}\right) .
\end{array}\right.
$$

Let $\mathbf{A}$ denote the map induced by system (3.8). Notice each entry of $\mathbf{A}$ is positive and it follows from (3.7) that the spectral radius of $\mathbf{A}$ is larger than unity. Since $x\left(t_{0}\right)=I_{a}\left(t_{0}\right)>0$ and $y\left(t_{0}\right)=I_{v}\left(t_{0}\right)>0$, solutions of (3.8) are unbounded. As a result, $I_{a}(t)$ and $I_{v}(t)$ also become unbounded large as $t \rightarrow \infty$. We obtain a contradiction and conclude that $W^{s}\left(\left\{E_{0}\right\}\right) \subset Y$. Therefore, system (2.2) is uniformly persistent with respect to $Y$ by [11, Theorem 4.1], i.e., there exists $m>$ 0 such that $\liminf _{t \rightarrow \infty} S_{a}(t) \geq m, \liminf _{t \rightarrow \infty} I_{a}(t) \geq m, \liminf _{t \rightarrow \infty} N_{a}(t) \geq$ $m$ and $\liminf _{t \rightarrow \infty} I_{v}(t) \geq m$ for any solution $\left(S_{a}(t), I_{a}(t), N_{a}(t), I_{v}(t)\right)$ with positive initial condition. The case when $p=1$ can be shown similarly using instability of $E_{0}$.

Although it is known that the crow family of birds have very high WNV mortality rate, the mortality rate of some other species of birds such as house barrow and common grackle are usually very small. In particular, European starling, rock dove, American robin, and several other species of birds have zero WNV mortality rate as shown in an experimental study by Komar [13]. Therefore, it is reasonable to consider the special case when there is no WNV related mortality for the avian population. In this situation $\lim _{t \rightarrow \infty} N_{a}(t)=$ $\Lambda_{a} / \mu_{a}$ and (2.2) has the following three-dimensional limiting system

$$
\left\{\begin{array}{l}
S_{a}(t+1)=\Lambda_{a}+\left(1-\mu_{a}\right) S_{a}(t)-\frac{b \beta_{a} \mu_{a}}{\Lambda_{a}} I_{v}(t) S_{a}(t) \\
I_{a}(t+1)=\frac{b \beta_{a} \mu_{a}}{\Lambda_{a}} I_{v}(t) S_{a}(t)+\left(1-\gamma_{a}-\mu_{a}\right) I_{a}(t) \\
I_{v}(t+1)=\left(1-\mu_{v}\right) I_{v}(t)+p \mu_{v} I_{v}(t)+\frac{b \beta_{v} \mu_{a}}{\Lambda_{a}} I_{a}(t)\left(N_{v}-I_{v}(t)\right) \\
S_{a}(0), I_{a}(0), I_{v}(0) \geq 0 .
\end{array}\right.
$$


Notice $R_{0}$ becomes

$$
R_{0}=\frac{b^{2} \mu_{a} N_{v} \beta_{a} \beta_{v}}{\Lambda_{a} \mu_{v}(1-p)\left(\gamma_{a}+\mu_{a}\right)} .
$$

We show that the disease-free equilibrium $\left(\Lambda_{a} / \mu_{a}, 0, \Lambda_{a} / \mu_{a}, 0\right)$ is globally asymptotically stable for (2.2) if $0 \leq p<1$ and $R_{0} \leq 1$.

Theorem 3.4. The disease-free equilibrium $E_{0}=\left(\Lambda_{a} / \mu_{a}, 0, \Lambda_{a} / \mu_{a}, 0\right)$ is the only equilibrium which is moreover globally asymptotically stable for system (2.2) if $\alpha_{a}=0,0 \leq p<1$, and $R_{0} \leq 1$.

Proof. It is clear that (2.2) has only the disease-free equilibrium. Since $S_{a}(t)+$ $I_{a}(t) \leq \Lambda_{a} / \mu_{a}$ and $I_{v}(t) \leq N_{v}$ for $t \geq 0$, we let

$$
\Delta=\left\{(x, y, z) \in \mathbb{R}_{+}^{3}: x+y \leq \frac{\Lambda_{a}}{\mu_{a}}, z \leq N_{v}\right\} .
$$

We construct a Liapunov function $V$

$$
V: \Delta \rightarrow \mathbb{R}_{+} \text {by } V\left(S_{a}, I_{a}, I_{v}\right)=\tilde{A}\left(\frac{\Lambda_{a}}{\mu_{a}}-S_{a}\right)+\tilde{B} I_{a}+\tilde{C} I_{v},
$$

where nonnegative $\tilde{A}, \tilde{B}$ and $\tilde{C}$ will be determined later. Let $G$ denote the map induced by system (3.9). Then $V \geq 0$ on $\Delta$ and

$$
\begin{aligned}
V\left(G\left(S_{a}, I_{a}, I_{v}\right)\right) \leq & \tilde{A}\left(1-\mu_{a}\right) \frac{\Lambda_{a}}{\mu_{a}}+\left[\tilde{B}\left(1-\gamma_{a}-\mu_{a}\right)+\tilde{C} \frac{b \beta_{v} \mu_{a}}{\Lambda_{a}} N_{v}\right] I_{a} \\
& +\left[\tilde{A} b \beta_{a}+\tilde{B} b \beta_{a}+\tilde{C}\left(1-\mu_{v}\right)+\tilde{C} p \mu_{v}\right] I_{v} .
\end{aligned}
$$

We choose $\tilde{A}=0$. Then $\tilde{B}$ and $\tilde{C}$ must satisfy

$$
\tilde{B}\left(1-\gamma_{a}-\mu_{a}\right)+\tilde{C} \frac{b \beta_{v} \mu_{a}}{\Lambda_{a}} N_{v} \leq \tilde{B} \quad \text { and } \quad \tilde{B} b \beta_{a}+\tilde{C}\left(1-\mu_{v}+p \mu_{v}\right) \leq \tilde{C} .
$$

We now let

$$
\tilde{C}=1 \quad \text { and } \quad \tilde{B}=\frac{b \beta_{v} \mu_{a} N_{v}}{\Lambda_{a}\left(\gamma_{a}+\mu_{a}\right)} .
$$

Then $\tilde{C}$ and $\tilde{B}$ clearly satisfy the above inequalities as $R_{0} \leq 1$. Hence $V\left(G\left(S_{a}\right.\right.$, $\left.\left.I_{a}, I_{v}\right)\right) \leq V\left(S_{a}, I_{a}, I_{v}\right)$ and $V$ is a Liapunov function on $\Delta$. 
Let $\mathcal{M}=\left\{\left(S_{a}, I_{a}, I_{v}\right) \in \Delta: V\left(G\left(S_{a}, I_{a}, I_{v}\right)\right)=V\left(S_{a}, I_{a}, I_{v}\right)\right\}$. Then $\mathcal{M}=$ $\left\{\left(S_{a}, I_{a}, I_{v}\right) \in \Delta: I_{a}=I_{v}=0\right\}$ and the only invariant set in $\mathcal{M}$ is $\left(\Lambda_{a} / \mu_{a}, 0,0\right)$. Therefore, $\left(\Lambda_{a} / \mu_{a}, 0,0\right)$ is globally asymptotically stable for system (3.9) by the LaSalle's invariance principle [8, 14]. Since the limiting system (3.9) has only one equilibrium which is moreover globally asymptotically stable when $R_{0} \leq 1$, applying [6], we conclude that the disease-free equilibrium is globally asymptotically stable for system (2.2) when $\alpha_{a}=0,0 \leq p<1$, and $R_{0} \leq 1$.

\section{Discussion}

It is showed in the previous section that the West Nile virus can be wiped out when $R_{0} \leq 1$ and $\mu_{a}=0$, and the disease can persist within the populations when $R_{0}>1$. Although it is proved that the disease-free equilibrium is globally asymptotically stable if $\mu_{a}=0,0 \leq p<1$ and $R_{0} \leq 1$, it is suspected that the disease-free equilibrium is globally asymptotically when $R_{0}<1, \mu_{a}>0$, and $0 \leq p<1$. Since stability analysis does not provide any information about the transient behavior of the model which may be very important in terms of eradication and management plans, we next use simple numerical methods to study (2.2).

To simulate model (2.2), we adopt the following parameter values: $\Lambda_{a}=140$, $\gamma_{a}=0.1, \alpha_{a}=0.1, \mu_{a}=0.02, b=0.7, \beta_{a}=1.0, \beta_{v}=0.38, \mu_{v}=0.06$, $N_{v}=200$ and $p=0.2$. Initial conditions are chosen to be $S_{a}(0)=1000$, $I_{a}(0)=0, N_{a}(0)=1000$ and $I_{v}(0)=100$ for all simulations presented. Notice in this case that $R_{0}=0.5038<1$ and system (2.2) has only the diseasefree equilibrium. Simulations for this set of parameter values are plotted in Figure 1(a). Both infected populations go to a peak at approximately the same time before they are diminished. Therefore there is a serge of the disease for a short period of time even when $R_{0}<1$.

We next keep the same parameter values but change $\beta_{v}$ from 0.38 to 0.78 . Then $R_{0}=1.0341>1$ and system (2.2) has a unique endemic equilibrium according to Theorem 3.3. The time evolution of the infected populations are plotted in Figure 1(b). It can be seen that both infected populations also increase before they decrease to the equilibrium levels for initial conditions with $I_{v}(0) \geq 10$. When $0<I_{v}(0)<10$, then both infected populations increase 
to equilibrium levels with increasing time. It is known that vertical transmission of virus in the vector population is an important factor for contributing the spread of the disease $[3,7,10]$. We shall investigate this factor using our built model. We vary the parameter value $p$ with the above fixed parameter values so that $R_{0}>1$. When $p=0.2$, it is calculated $R_{0}=1.0341$, when $p=0.3$, $R_{0}=1.1818$, and $R_{0}=1.3788$ when $p=0.4$. The resulting time series of the infected mosquitoes and birds are plotted in Figure 1 (c) and (d) respectively. We see from these two plots that increasing the vertical transmission rate $p$ increases the equilibrium levels and hence increases severity of the epidemics as the peaks of infectives increase with increasing $p$. However, the time that these peaks occurred is approximately independent of $p$.

In this manuscript, a simple West Nile epidemic model in discrete-time is proposed and analyzed. Our modeling assumptions are based on a continuous-time model developed by Cruz-Pacheco et al. [5]. In particular, the avian population in the absence of the disease is stabilized in a constant population level and the transmission of the virus is either through infected mosquito bites or natural birth of infected vectors. The dynamics of the epidemics depend on a lumped parameter $R_{0}$. The disease-free equilibrium $E_{0}$ is the only equilibrium and is locally asymptotically stable if $R_{0}<1$. It is proved that $E_{0}$ is globally asymptotically stable when there is no disease related mortality for the avian population and $R_{0} \leq 1$. As a result, the disease can be wiped out in this special situation. However, the epidemic can persist if $R_{0}>1$. From the data given in $[5,16]$, it is very often that $R_{0}>1$ for many species of birds along with vertical transmission of the vector population. Therefore, very likely that the West Nile epidemic can persist in natural populations as it has been observed in recent years in the U.S.

It is demonstrated numerically via simulations that both infected populations increase initially even when $R_{0}<1$ and the transient behavior of the model depends on initial conditions when $R_{0}>1$. If the initial infected vector population is small, then both infected populations will increase until they reach the equilibrium levels. However, if the initial infected vector population is large, then both infected vector and host populations will reach a maximum number which is much larger than the equilibrium value in a short period of time before they decrease to the equilibrium levels as shown in Figure 1. Therefore in 
this situation there will be a severe outbreak of the disease in the beginning of the epidemic.

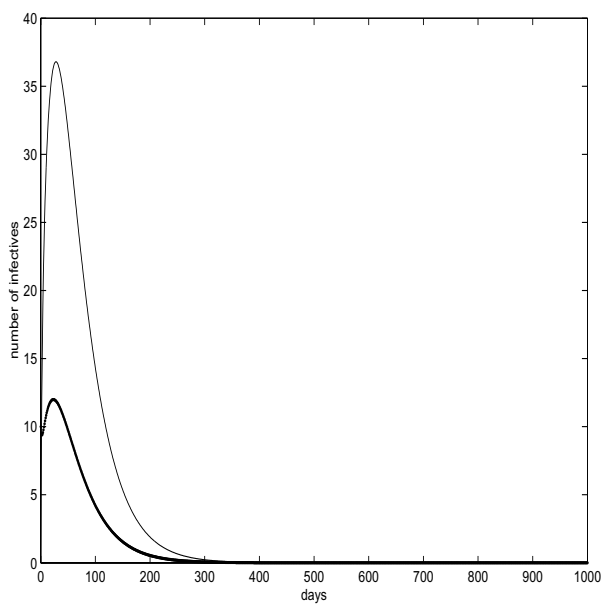

(a)

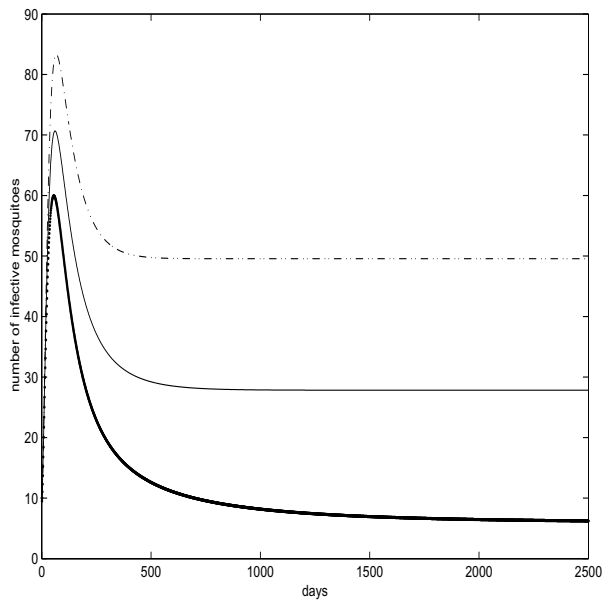

(c)

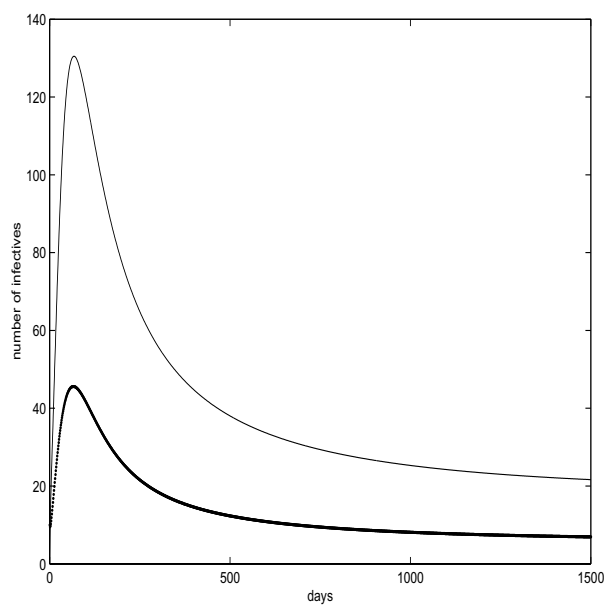

(b)

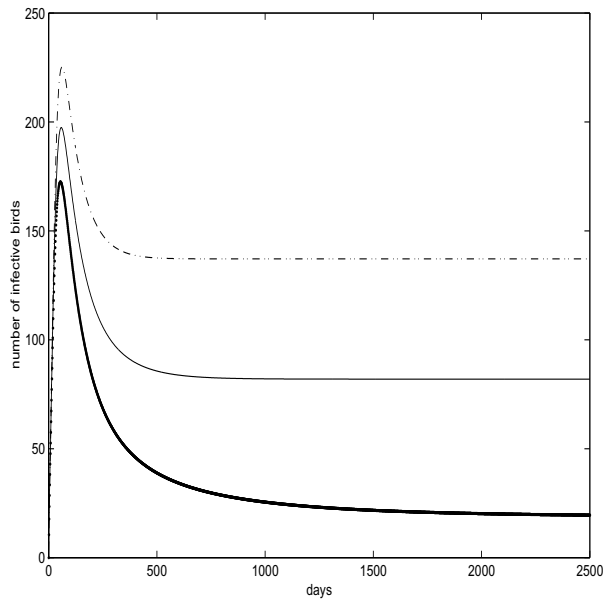

(d)

Figure 1 - (a) and (b) plot infective populations when $R_{0}<1$ and $R_{0}>1$, respectively. The solid lines are for the mosquito population and the dotted lines are for the bird population. (c) and (d) plot number of infective mosquitoes and birds versus time for different values of $p$, respectively. Solid lines are for $p=0.2$, dotted lines are for $p=0.3$, and dash-dotted lines are for $p=0.4$. 


\section{REFERENCES}

[1] L. Allen, Some discrete-time SI, SIR and SIS epidemic models. Math. Biosci., 124 (1994), 83-97.

[2] L. Allen, Comparison of deterministic and stochastic SIS and SIR models in discrete time. Math. Biosci., 163 (2000), 1-33.

[3] S. Baqar, C. Hayes, J. Murphy and D. Watts, Vertical transmission of West Nile Virus by Culex and Aedes species mosquitoes. Am. J. Trop. Med. Hyg., 48 (2002), 757-762.

[4] http://www.cdc.gov/ncidod/dvbid/westnile

[5] G. Cruz-Pacheco, L. Esteva, J. Montano-Hirose and C. Vargas, Modelling the dynamics of West Nile virus. Bull. Math. Biol., 67 (2005), 1157-1172.

[6] J.M. Cushing, A strong ergodic theorem for some nonlinear matrix models for the dynamics of structured population. Natural Resources Modeling, 3 (1989), 331-357.

[7] D. Dohm, M. Sardelis and M. Turell, Experimental vertical transmission of West Nile Virus by Culex pipiens. J. Med. Entomol., 39 (2002), 640-644.

[8] S. Elaydi, An Introduction to Difference Equations. Second Edition, Springer, New York, 1999.

[9] J. Franke and A. Yakubu, Discrete-time SIS epidemic models in a seasonal environment. SIAM Appl. Math., 66 (2006), 1563-1587.

[10] L. Goddard, A. Roth, W. Reisen and T. Scott, Vertical transmission of West Nile Virus by three California Culex species. J. Med. Entomol., 40 (2003), 743-746.

[11] J. Hofbauer and J. So, Uniform persistence and repellors for maps. Proc. Am. Math. Soc., 107 (1989), 1129-1142.

[12] W.O. Kermack and A.G. McKendrick, A contribution to the mathematical theory of epidemics. Proc. Roy. Soc. London Ser. A, 115 (1927), 700-721.

[13] N. Komar, WNV vertebrate ecology and biology-birds, 2003. www.cdc.gov/ncidod/dvbid/ westnile/conf/pdf/Komar4th03.pdf.

[14] J.P. LaSalle, The Stability of Dynamical Systems. SIAM, 1976.

[15] M.A. Lewis, J. Renclawowicz, P. van Den Driessche and W. Marjorie, A comparison of continuous and discrete time West Nile virus models. Bull. Math. Bio., 68 (2006), 491-509.

[16] http://www.animaldiversity.ummz.umich.edu. The University of Michigan Museum of Zoology.

[17] D. Thomas and B. Urena, A model describing the evolution of West-Nile like encephalities in New York city. Math. Comput. Model., 34 (2001), 771-781.

Comp. Appl. Math., Vol. 26, N. 3, 2007 